$4 / 1 / 19485(2)$

PREPARED FOR THE U.S. DEPARTMENT OF ENERGY, UNDER CONTRACT DE-AC02-76-CHO-3073
PPPL-3024

UC-420,427
DIRECT VARIATIONAL SOLUTIONS TO

THE GRAD-SCHLUTTER-SHAFRANOV EQUATION

BY

G.O. LUDWIG

NOVEMBER 1994
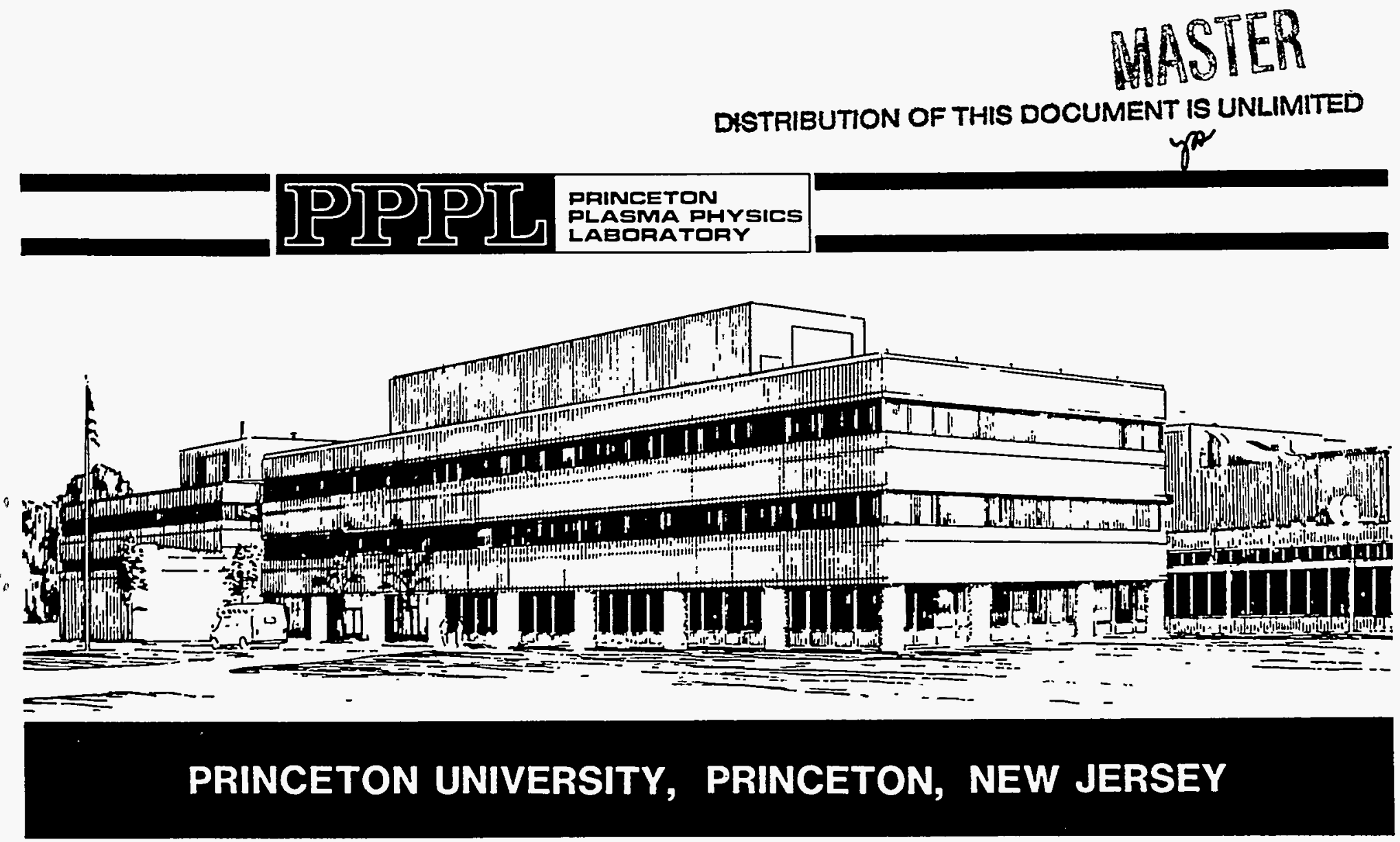


\section{NOTICE}

This report was prepared as an account of work sponsored by an agency of the United States Government. Neither the United States Government nor any agency thereof, nor any of their employees, makes any warranty, express or implied, or assumes any legal liability or responsibility for the accuracy, completeness, or usefulness of any information, apparatus, product, or process disclosed, or represents that its use would not infringe privately owned rights. Reference herein to any specific commercial produce, process, or service by trade name, trademark, manufacturer, or otherwise, does not necessarily constitute or imply its endorsement, recommendation, or favoring by the United States Government or any agency thereof. The views and opinions of authors expressed herein do not necessarily state or reflect those of the United States Government or any agency thereof.

\section{NOTICE}

This report has been reproduced from the best available copy.

Available in paper copy and microfiche.

Number of pages in this report: 24

DOE and DOE contractors can obtain copies of this report from:

Office of Scientific and Technical Information

P.O. Box 62

Oak Ridge, TN 37831 ;

(615) 576-8401.

This report is publicly available from the:

National Technical Information Service

Department of Commerce

5285 Port Royal Road

Springfield, Virginia 22161

(703) $487-4650$ 


\section{DISCLAIMER}

Portions of this document may be illegible in electronic image products. Images are produced from the best available original document. 


\title{
Direct Variational Solutions to the Grad-Schlüter-Shafranov Equation
}

\author{
G O Ludwig* \\ Princeton Plasma Physics Laboratory \\ P O Box 451, Princeton, New Jersey 08.543
}

7 September 1994

\begin{abstract}
A direct variational method based on a energy principle is applied to obtain approximate magnetohydrodynamic equilibria for tokamak plasmas. The geometry of the nested magnetic flux surfaces is specified by a model that includes displacement, elongation and triangularity effects. The radial dependence in flux coordinates is described by a set of consistent trial functions which allows analytical calculation of the flux-surface averaged internal energy density of the plasma. Approximate solutions of the variational problem are obtained for arbitrary aspect-ratio tokamaks using a one-parameter optimization procedure.
\end{abstract}

* Permanent $\lambda$ dddress: Instituto Nacional de Pesquisas Espaciais - INPE, Caixa Postal 515, 12201-970 - São José dos Campos, São Paulo, Brazil. Work partially supported by the Fundação de Amparo à Pesquisa do Estado de São Paulo - FAPESP. 


\section{Introduction}

The magnetohydrodynamic (MHD) equilibrium of an axisymmetric toroidal plasma can be described by variational principles, either in the energy form [1] or in the Lagrangian form [2]. Lsually these variational problems are not solved directly, but by reducing the problem to one involving a quasilinear elliptic partial differential equation, the Grad-Schlüter-Shafranov equation [3][4][5], or a system of coupled, nonlinear, ordinary differential equations as in the function parametrization method [6] or in the variational moment method [ 7$]$. These differential equations can be solved analytically only in a small neighborhood of the magnetic axis, i.e., using the small toroidicity expansion, or for some special geometries, or. in general, by numerical methods. However, to find an approximate solution over the plasma cross-section, with prescribed boundary conditions on the plasma edge, it can be advantageous to use direct variational methods.

The direct method reported here uses simple analytical expressions for the description of the flux surface geometry which are valid for arbitrary aspectratio. The approximate solutions generated by the method allow straightforward calculation of all the relevant equilibrium parameters and profiles. These solutions should be suitable for transport, current drive and stability studies. The accuracy of the solutions can be improved by a sequence of trial functions of increasing complexity and with increasing number of free parameters. An attractive characteristic of the method is the possibility of obtaining fast approximate equilibrium solutions with modest computational resources.

In section 2 the variational problem is formulated in a manner suitable for direct methods of solution. A consistent set of trial functions is constructed in section 3 starting from a Taylor series expansion near the magnetic axis. The expressions derived there correspond to a truncated Fourier series expansion for the inverse mapping which includes displacement, elongation and triangularity effects (the triangularity approximation). In section 4 the flux-surface averaged coefficients in the equilibrium equation are evaluated in terms of the trial functions. Finally, using a one-parameter optimization procedure approximate solutions for the equilibrium of two different tokamak configurations are presented in section 5 , with a brief discussion about possible extensions of the method. 


\section{Variational Principle}

Consider the functional

$$
Q(a)=\iiint_{V(a)} d^{3} r \mathcal{L}\left(\Phi_{P}, \nabla \Phi_{P}, h_{\zeta}\right)
$$

where $a$ denotes the plasma boundary, $\Phi_{P}$ is the poloidal flux in an axisymmetric toroidal plasma equilibrium, $\zeta$ is the toroidal symmetry angle and $h_{\zeta}=|\partial \vec{r} / \partial \zeta|$ is the distance to the symmetry axis. The Lagrangian density is defined by

$$
\mathcal{L}=\frac{B_{P}^{2}}{2 \mu_{0}}-\frac{B_{T}^{2}}{2 \mu_{0}}-p
$$

where $p\left(\Phi_{P}\right)$ is the plasma pressure and

$$
\begin{aligned}
& B_{P}=\left|\nabla \Phi_{P}\right| /\left(2 \pi h_{\zeta}\right), \\
& B_{T}=\mu_{0} I\left(\Phi_{P}\right) /\left(2 \pi h_{\zeta}\right)
\end{aligned}
$$

are, respectively, the poloidal and the toroidal components of the induction. Here $I\left(\Phi_{P}\right)$ is the total poloidal current between the symmetry axis and a given magnetic surface. For a fixed boundary condition $\delta \Phi_{P}(a)=0$, the extremum of $Q(a)$ is reached for that function $\Phi_{P}$ which is a solution of the Grad-Schlüter-Shafranov equation [6]

$$
\triangle^{2} \Phi_{P}=2 \pi \mu_{0} h_{\zeta} j_{T}
$$

where

$$
j_{T}=-2 \pi h_{\zeta} \frac{d p}{d \Phi_{P}}-\frac{\mu_{0} I}{2 \pi h_{\zeta}} \frac{d I}{d \Phi_{P}}
$$

is the toroidal component of the current density.

In flux coordinates $(\rho, \theta, \zeta)$ the functional becomes

$$
Q(a)=\int_{0}^{a} d \rho\langle\mathcal{L}\rangle\left(\Phi_{P}, d \Phi_{P} / d \rho, \rho\right)
$$

where

$$
\langle\mathcal{L}\rangle=\frac{K(\rho)}{2}\left(\frac{d \Phi_{P}}{d \rho}\right)^{2}-\frac{I^{2}\left(\Phi_{P}\right)}{2} \frac{d L}{d \rho}-p\left(\Phi_{P}\right) \frac{d V}{d \rho}
$$


is the flux-surface averaged Lagrangian density and the coefficients

$$
\begin{aligned}
V(\rho) & =2 \pi \iint_{S_{T}} h_{\zeta} d^{2} r(\zeta), \\
L(\rho) & =\frac{\mu_{0}}{2 \pi} \iint_{S_{T}} \frac{d^{2} r(\zeta)}{h_{\zeta}}, \\
K(\rho) & =\frac{1}{2 \pi \mu_{0}} \oint \frac{|\nabla \rho|}{h_{\zeta}} d \ell_{\theta},
\end{aligned}
$$

give, respectively, the volume enclosed by a magnetic surface, the inductance of the toroidal solenoid which coincides with a given flux surface, and the inverse kernel to calculate the self-inductance of the plasma loop [8].

Let $\Phi_{P}$ denote a generalized coordinate so that the toroidal plasma current through the poloidal cross-section $S_{T}(\rho)$ of a magnetic flux surface,

$$
I_{T}(\rho)=K(\rho) d \Phi_{P} / d \rho
$$

represents the generalized momentum. Then, the total energy

$$
W(a)=\iiint_{V(a)} d^{3} r\left(\frac{B_{P}^{2}}{2 \mu_{0}}+\frac{B_{T}^{2}}{2 \mu_{0}}+p\right)=\int_{0}^{a} d \rho\langle\mathcal{H}\rangle\left(\Phi_{P}, I_{T}, \rho\right)
$$

is given in terms of the flux-surface averaged Hamiltonian density

$$
\langle\mathcal{H}\rangle=\frac{I_{T}^{2}(\rho)}{2 K(\rho)}+\frac{I^{2}\left(\Phi_{P}\right)}{2} \frac{d L}{d \rho}+p\left(\Phi_{P}\right) \frac{d V}{d \rho} .
$$

Now, using the canonical system of Euler equations for the functional $Q(\rho$, $\Phi_{P}, I_{\mathcal{T}}$ and $\langle\mathcal{H}\rangle$ are the canonical variables), it is easy to show that $W(a)$ is stationary under the transformation of the topological radius

$$
\rho^{*}=\rho+\xi,
$$

where the virtual displacement $\xi$ obeys the boundary conditions

$$
\xi(0)=\xi(a)=0 .
$$

Integrating the second term in the expression for $\langle\mathcal{H}\rangle$ by parts, one can separate the contribution of the magnetostatic energy due to the external induction $B_{T, 0}$,

$$
W_{T, 0}(a)=\iiint_{V(a)} d^{3} \rho \frac{B_{T, 0}^{2}}{2 \mu_{0}}=\frac{1}{2} L(a) I^{2}(a) .
$$


This contribution of the vacuum field does not involve the plasma itself and can be omitted in the course of the variation for a fixed boundary problem. Hence one can define the internal energy of the plasma

$$
U(a)=W_{P}(a)+W_{T}(a)+W_{t h}(a),
$$

where

$$
\begin{aligned}
W_{P}(a) & =\iiint_{V(a)} d^{3} r \frac{B_{P}^{2}}{2 \mu_{0}}=\int_{0}^{a} d \rho \frac{I_{T}^{2}(\rho)}{2 K(\rho)}, \\
W_{T}(a) & =\iiint_{V(a)} d^{3} r \frac{B_{T}^{2}-B_{T}^{2}, 0}{2 \mu_{0}}=-\int_{0}^{a} d \rho L(\rho) I(\rho) \frac{d I}{d \rho}, \\
W_{t h}(a) & =\iiint_{V(a)} d^{3} r p=\int_{0}^{a} d \rho p(\rho) \frac{d V}{d \rho}
\end{aligned}
$$

are, respectively, the internal magnetostatic energy stored in the plasma loop, the internal magnetostatic energy stored in the plasma solenoid and the thermal energy of the plasma. Using the equilibrium equation (flux-surface averaged Grad-Schlüter-Shafranov equation)

$$
\frac{d \Phi_{P}}{d \rho} \frac{d}{d \rho}\left(K(\rho) \frac{d \Phi_{P}}{d \rho}\right)=-\frac{d L}{d \rho} I(\rho) \frac{d I}{d \rho}-\frac{d V}{d \rho} \frac{d p}{d \rho}
$$

the total poloidal current $I(\rho)$ is eliminated in terms of the toroidal plasma current $I_{T}(\rho)$ :

$$
W_{T}(a)=\int_{0}^{a} d \rho \frac{L(\rho)}{d L / d \rho}\left(\frac{I_{T}(\rho)}{K(\rho)} \frac{d I_{T}}{d \rho}+\frac{d V}{d \rho} \frac{d p}{d \rho}\right) .
$$

The variational problem consists in finding stationary values of the internal energy $U(a)$, under virtual displacements, for given profiles $I_{T}(\rho)$, $p(\rho)$ and fixed values of the total toroidal plasma current $I_{T}(a)$ and plasma pressure at the magnetic axis $p(0)$. It remains to evaluate the flux-surface averaged coefficients $V(\rho), L(\rho)$ and $K(\rho)$ in the equilibrium equation in terms of the plasma geometry. For completeness, the following integral relations for the toroidal magnetic flux $\Phi_{T}(\rho)$. the safety factor $q(\rho)$, the poloidal plasma 
current $I_{P}(a)$ and the total poloidal current $I(\rho)$ are presented:

$$
\begin{aligned}
\Phi_{T}(\rho) & =\int_{0}^{\rho} d \rho^{\prime} I\left(\rho^{\prime}\right) \frac{d L}{d \rho^{\prime}} \\
q(\rho) & =\frac{d \Phi_{T} / d \rho}{d \Phi_{P} / d \rho}=\frac{I(\rho)}{I_{T}(\rho)} K(\rho) \frac{d L}{d \rho} \\
I_{P}(\rho) & =I(0)-I(\rho) \\
I(\rho) & =\left[I^{2}(a)+\int_{\rho}^{a} d \rho^{\prime} \frac{2}{d L / d \rho^{\prime}}\left(\frac{I_{T}\left(\rho^{\prime}\right)}{K^{\prime}\left(\rho^{\prime}\right)} \frac{d I_{T}}{d \rho^{\prime}}+\frac{d V}{d \rho^{\prime}} \frac{d p}{d \rho^{\prime}}\right)\right]^{1 / 2}
\end{aligned}
$$

where

$$
I(a)=2 \pi R_{0}(a) B_{0} / \mu_{0}
$$

and $B_{0}$ is the external magnetic flux density at the geometric center $R_{0}(a)$ of the plasma cross-section. On the magnetic axis one has

$$
I(0)=2 \pi R_{m} B_{m} / \mu_{0}
$$

where $R_{m}$ is the distance to the axis of symmetry and $B_{m}$ gives the induction on the magnetic axis.

Finally, using a normalization appropriate for the low toroidicity limit $\rho \rightarrow 0$, one defines the internal inductance $\ell_{i}$. the current diamagnetism $\mu_{I}$ and the current beta $\beta_{I}$ in terms of the energy- content of the plasma:

$$
\begin{aligned}
\ell_{i} & =4 W_{P}(a) /\left(\mu_{0} R_{m} I_{T}^{2}(a)\right), \\
\mu_{I} & =4 W_{T}(a) /\left(\mu_{0} R_{m} I_{T}^{2}(a)\right), \\
\beta_{I} & =4 W_{t h}(a) /\left(\mu_{0} R_{m} I_{T}^{2}(a)\right),
\end{aligned}
$$

where the total toroidal plasma current is given by

$$
I_{T}(a)=\frac{1}{\mu_{0}}\left(\oint B_{P} d \varepsilon_{\theta}\right)_{a} .
$$

The internal self-inductance of the plasma loop is

$$
L_{T}(a)=\frac{\mu_{0} R_{m}}{2} \ell_{i} .
$$




\section{Trial Functions}

A set of consistent trial functions which describes the plasma geometry can be obtained from a Taylor series expansion of the poloidal flux near the magnetic axis. For an axisymmetric and up-down symmetric MHD equilibrium, a third order expansion in cylindrical coordinates gives

$$
\begin{aligned}
\psi(R, Z) \cong & \frac{\left(R-R_{m}\right)^{2}}{2} \psi^{(2,0)}\left(R_{m}, 0\right)+\frac{Z^{2}}{2} \psi^{(0,2)}\left(R_{m}, 0\right) \\
& +\frac{\left(R-R_{m}\right)^{3}}{6} \psi^{(3,0)}\left(R_{m}, 0\right)+\frac{Z^{2}\left(R-R_{m}\right)}{2} \psi^{(1,2)}\left(R_{m}, 0\right),
\end{aligned}
$$

where $\psi=\Phi_{P} / \Phi_{P}(a)$ is the normalized poloidal flux function and $\left(R_{m}, 0\right)$ corresponds to the position of the magnetic axis. Consistently with this approximation one represents the transformation to flux coordinates $(R, Z) \rightarrow$ $(\rho, \theta)$ by the truncated Fourier series expansions [9]

$$
\begin{aligned}
& R(\rho . \theta) \cong R_{0}(\rho)-\frac{\rho T(\rho)}{4}+\rho \cos \theta+\frac{\rho T(\rho)}{4} \cos 2 \theta, \\
& Z(\rho, \theta) \cong \rho E(\rho)\left(\sin \theta-\frac{T(\rho)}{4} \sin 2 \theta\right),
\end{aligned}
$$

where $R_{0}(\rho)$ represents the geometric centers of the flux surfaces, $T(\rho)$ is the triangularity coefficient and $E(\rho)$ is the elongation coefficient. On the magnetic axis one has $R_{0}(0)=R_{m}$ and $E(0)=E_{m}$. The small toroidicity expansion gives:

$$
\begin{aligned}
R_{0}(\rho) & \cong R_{m}+\rho R_{0}^{\prime}(0)+\rho^{2} R_{0}^{\prime \prime}(0) / 2+\mathcal{O}[\rho]^{3}, \\
E(\rho) & \cong E_{m}+\rho E^{\prime}(0)+\mathcal{O}[\rho]^{2} \\
T(\rho) & \cong T(0)+\rho T^{\prime}(0)+\mathcal{O}[\rho]^{2}
\end{aligned}
$$

The corresponding truncated Fourier series expansions become:

$$
\begin{aligned}
R(\rho, \theta) \cong & R_{m}+\rho\left(R_{0}^{\prime}(0)-\frac{T(0)}{4}+\cos \theta+\frac{T(0)}{4} \cos 2 \theta\right) \\
& +\frac{\rho^{2}}{2}\left(R_{0}^{\prime \prime}(0)-\frac{T^{\prime}(0)}{2}+\frac{T^{\prime}(0)}{2} \cos 2 \theta\right)+\mathcal{O}[\rho]^{3}, \\
Z(\rho, \theta) \cong & \rho E_{m}\left(\sin \theta-\frac{T(0)}{4} \sin 2 \theta\right) \\
& +\rho^{2}\left(E^{\prime}(0) \sin \theta-E_{m} \frac{T^{\prime}(0)}{4} \sin 2 \theta-E^{\prime}(0) \frac{T(0)}{4} \sin 2 \theta\right)+\mathcal{O}[\rho]^{3} .
\end{aligned}
$$


Furthermore, the small toroidicity expansion of $\psi(\rho)$ to third order is

$$
\psi(\rho) \cong \frac{\rho^{2}}{2 !} \psi^{\prime \prime}(0)+\frac{\rho^{3}}{3 !} \psi^{\prime \prime \prime}(0)+\mathcal{O}[\rho]^{4}
$$

Substituting the Fourier series expansions for $R(\rho, \theta)$ and $Z(\rho, \theta)$ in the Taylor series expansion for $\psi(R, Z)$ one obtains the consistency relations:

$$
\begin{aligned}
R_{0}^{\prime}(0) & =0 \\
E_{m}^{2} \psi^{(0,2)} & =\psi^{(2.0)} \\
T(0) & =0 \\
R_{0}^{\prime \prime}(0) & =-\psi^{(3.0)} /\left(3 \psi^{(2,0)}\right) . \\
E^{\prime}(0) & =0 \\
T^{\prime}(0) & =\left(3 E_{m}^{2} \psi^{(1,2)}-\psi^{(3,0)}\right) /\left(6 \psi^{(2.0)}\right) .
\end{aligned}
$$

A complete power series solution can be found by substituting the expansions for $R, Z$ and $\psi$ in the Grad-Schlüter-Shafranov equation. One finds, firstly, that $p(\rho)$ and $I(\rho)$ must be functions of $\rho^{2}$, i.e., must have zero odd derivatives on the magnetic axis for the assumed symmetry. Secondly, that the coefficient $\psi^{(2,0)}$ can be eliminated in order to cancel the leading order term of the equation. The expression for $\psi(\rho)$ immediately yields

$$
\psi(\rho) \cong \frac{E_{m}\left[-\mu_{0}^{2} I(0) I^{\prime \prime}(0)-4 \pi^{2} \mu_{0} R_{m}^{2} p^{\prime \prime}(0)\right]^{1 / 2}}{2\left(E_{m}^{2}+1\right)^{1 / 2}} \rho^{2}+\mathcal{O}[\rho]^{4} .
$$

Next, the coefficient $\psi^{(3.0)}$ is calculated in order to cancel the remaining term of the Grad-Schlüter-Shafranov equation and the coefficient $\psi^{(1,2)}$ is evaluated in terms of the triangularity $T(a)$ at the plasma edge. Finally, $R_{m}$ is evaluated in terms of $R_{0}(a)$, the geometric center of the plasma crosssection, and $E_{m}$ in terms of the elongation $E(a)$ at the plasma edge (assuming always the validity of the small toroidicity approximation).

A different approach, that will be pursued in this paper, is to consider the expansions of the Fourier amplitudes as trial functions in a variational calculation valid for arbitrary aspect-ratio. Extending the expansions of the Fourier amplitudes up to the plasma edge one obtains the following trial functions in the triangularity approximation:

$$
\begin{aligned}
R_{0}(\rho) & \cong R_{m}-\left[R_{m}-R_{0}(a)\right](\rho / a)^{2}, \\
E(\rho) & \cong E_{m} \cong E(a), \\
T(\rho) & \cong T(a)(\rho / a) .
\end{aligned}
$$


In this context the truncated Fourier expansions $R(\rho, \theta), Z(\rho, \theta)$ for the inverse mapping are taken as parametric equations for the self-similar flux surfaces. One can immediately write the expressions for the partial derivatives:

$$
\begin{aligned}
& \partial R / \partial \rho \cong R_{0}^{\prime}+\cos \theta-T \sin ^{2} \theta \\
& \partial Z / \partial \rho \cong E_{m}(1-T \cos \theta) \sin \theta \\
& \partial R / \partial \theta \cong-\rho(1+T \cos \theta) \sin \theta \\
& \partial Z / \partial \theta \cong \rho E_{m}\left(\cos \theta+T / 2-T \cos ^{2} \theta\right) .
\end{aligned}
$$

Note that the coefficients of triangularity $T(a)$ and of elongation $E(a)$ at the plasma edge are related to the geometric triangularity $\delta(a)$ and to the geometric elongation $\kappa(a)$ by

$$
\begin{aligned}
T(a)= & \frac{8}{3} \delta(a)-4 \sqrt{2} \sqrt{1+\frac{2}{9} \delta^{2}(a)} \\
& \times \sin \left[\frac{1}{3} \arctan \left(\frac{\sqrt{3}\left[135-16 \delta^{2}(a)\right] \delta(a)}{27 \sqrt{96-11 \delta^{2}(a)+32 \delta^{4}(a)}}\right)\right], \\
E(a)= & \kappa(a) \frac{4}{3+\sqrt{1+2 T^{2}(a)}} \frac{2 T(a)}{\sqrt{2 \sqrt{1+T^{2}(a)}-2+2 T^{2}(a)}}
\end{aligned}
$$

The inverse relations for arbitrary radius $\rho$ are:

$$
\begin{aligned}
& \delta(\rho)=\frac{3\left(\sqrt{1+2 T^{2}(\rho)}-1\right)+T^{2}(\rho)}{4 T(\rho)} \\
& \kappa(\rho)=E(\rho) \frac{3+\sqrt{1+2 T^{2}(\rho)}}{4} \frac{\sqrt{2 \sqrt{1+2 T^{2}(\rho)}-2+2 T^{2}(\rho)}}{2 T(\rho)} .
\end{aligned}
$$

For small $T(\rho)$, clearly, $\delta(\rho) \cong T(\rho)$ and $\kappa(\rho) \cong E(\rho)$.

\section{Flux-surface Averaged Coefficients}

The coefficients $V(\rho), L(\rho)$ and $K(\rho)$ can be evaluated in a straightforward manner using the trial functions derived in the previous section. It is useful, in the first place, to evaluate the area of the poloidal cross-section (the integrations carried out here are exact; the approximation corresponds to the 
use of trial functions)

$$
S_{T}(\rho)=\int_{0}^{2 \pi}\left(R \frac{\partial Z}{\partial \theta}\right) d \theta \cong \pi \rho^{2} E_{m}\left(1-\frac{T^{2}}{8}\right) .
$$

The volume enclosed by a magnetic surface is

$$
V(\rho)=\pi \int_{0}^{2 \pi}\left(R^{2} \frac{\partial Z}{\partial \theta}\right) d \theta \cong 2 \pi\left(R_{0}-\frac{\rho T}{4}\right) S_{T} .
$$

Introducing the local aspect-ratio

$$
A(\rho)=R_{0}(\rho) / \rho
$$

the relevant metric coefficients in the transformation $(R, Z) \rightarrow(\rho, \theta)$ to curvilinear coordinates are given by

$$
\begin{aligned}
h_{\zeta}= & R \cong \rho\left(A-\frac{T}{2}+\cos \theta+\frac{T}{2} \cos ^{2} \theta\right), \\
h_{\theta}^{2}= & \left(\frac{\partial R}{\partial \theta}\right)^{2}+\left(\frac{\partial Z}{\partial \theta}\right)^{2} \cong \rho^{2}\left[1+\frac{E_{m}^{2} T^{2}}{4}+\left(E_{m}^{2}+2\right) T \cos \theta\right. \\
& \left.+\left(E_{m}^{2}-1\right)\left(1-T^{2}\right) \cos ^{2} \theta-2\left(E_{m}^{2}+1\right) T \cos ^{3} \theta+\left(E_{m}^{2}-1\right) T^{2} \cos ^{4} \theta\right] .
\end{aligned}
$$

The Jacobian $\sqrt{g}$ can be obtained from

$$
\begin{aligned}
\frac{\sqrt{g}}{h_{\zeta}} & =\frac{\partial R}{\partial \rho} \frac{\partial Z}{\partial \theta}-\frac{\partial R}{\partial \theta} \frac{\partial Z}{\partial \rho} \\
& \cong \rho E_{m}\left[1+\frac{R_{0}^{\prime} T}{2}-\frac{T^{2}}{2}+\left(R_{0}^{\prime}-\frac{T}{2}\right)\left(\cos \theta-T \cos ^{2} \theta\right)\right] .
\end{aligned}
$$

Since $\sqrt{g} / h_{\zeta}$ is real and positive one verifies that the coefficient of triangularity is restricted to the range $-2<T(\rho)<2$ and that the rate of increase of the Shafranov shift $R_{0}^{\prime}(\rho)$ must satisfy the conditions:

$$
\begin{array}{ccc}
-1<R_{0}^{\prime}<-\frac{\left(7-4 T^{2}\right) T}{2\left(1+2 T^{2}\right)} & \text { for } & -2<T<-\frac{1}{2} \\
-1<R_{0}^{\prime}<1 & \text { for } & -\frac{1}{2}<T<\frac{1}{2} \\
-\frac{\left(7-4 T^{2}\right) T}{2\left(1+2 T^{2}\right)}<R_{0}^{\prime}<1 & \text { for } & \frac{1}{2}<T<2 .
\end{array}
$$


Hence one may write conditions for maximum positive Shafranov shift and plasma cross-sections of positive triangularity as follows:

$$
\begin{array}{cl}
\frac{R_{m}-R_{0}(a)}{a}<\frac{1}{2} & \text { for } \quad 0<T(a)<\frac{1}{2}, \\
\frac{R_{m}-R_{0}(a)}{a}<\frac{\left[7-4 T^{2}(a)\right] T(a)}{4\left[1+2 T^{2}(a)\right]} & \text { for } \quad \frac{1}{2}<T(a)<\frac{\sqrt{7}}{2} .
\end{array}
$$

Furthermore, since $h_{\zeta}$ is also a real positive quantity and $A>1$. the triangularity coefficient must satisfy the constraint (the plasma edge is tangent to the symmetry axis at the upper limit of this inequality)

$$
T<A+\sqrt{A^{2}-1} .
$$

Thus one may write in general

$$
-2<T<\min \left[2, A+\sqrt{A^{2}-1}\right] .
$$

If $A>5 / 4$ and $T=2$ the plasma cross-section described by the parametric equations in the triangularity approximation presents three cusps. However, if $0<T<1$ the cross-section is always D-shaped, which is the configuration of interest.

Now, using partial fractions decomposition, the flux-surface integral for the inductance coefficient $L(\rho)$ becomes

$$
\begin{aligned}
L(\rho)= & -\frac{\mu_{0}}{2 \pi} \int_{0}^{2 \pi}\left(\frac{Z}{R} \frac{\partial R}{\partial \theta}\right) d \theta \cong \frac{\mu_{0} \rho E_{m}}{\pi} \int_{0}^{\pi}\left(T \cos ^{2} \theta-3 \cos \theta+\frac{4}{T}-2 A\right. \\
& \left.+\frac{6+2 A(2 A-T-4 / T)+2(5 A-T-4 / T) \cos \theta}{2 A-T+2 \cos \theta+T \cos ^{2} \theta}\right) d \theta
\end{aligned}
$$

which results in the final form

$$
\frac{L(\rho)}{\mu_{0} \rho E_{m}} \cong \frac{T}{2}+\frac{4}{T}-2 A-\frac{4-\left(5 A-3 \sqrt{A^{2}-1}\right) T+T^{2}}{T \sqrt{1-T\left(A-\sqrt{A^{2}-1}\right)}} .
$$

In the limit $T \rightarrow 0$, that is, for a plasma of elliptical cross-section, one obtains

$$
\frac{L(\rho)}{\mu_{0} \rho E_{m}} \underset{T \rightarrow 0}{\rightarrow} A-\sqrt{A^{2}-1} \underset{\rho \rightarrow 0}{\rightarrow} \frac{\rho}{2 R_{m}} .
$$


Similarly, the inverse kernel $K(\rho)$ of the internal self-inductance is given by the integral

$$
\begin{aligned}
K(\rho)= & K\left(A, E_{m}, T, R_{0}^{\prime}\right)=\frac{1}{2 \pi \mu_{0}} \int_{0}^{2 \pi} \frac{h_{\theta}^{2} d \theta}{\sqrt{g}} \\
\cong & \frac{1}{\pi \mu_{0} E_{m}} \int_{0}^{\pi} d \theta\left[\frac{4\left(E_{m}^{2}-1\right)}{-2 R_{0}^{\prime}+T}+\frac{C_{1}+(A+\cos \theta) C_{2}}{D\left(2 A-T+2 \cos \theta+T \cos ^{2} \theta\right)}\right. \\
& \left.+\frac{4-T^{2}}{-2 R_{0}^{\prime}+T} \frac{C_{3}+\left[2-R_{0}^{\prime} T+\left(2 R_{0}^{\prime}-T\right) \cos \theta\right] C_{4}}{D\left[2+R_{0}^{\prime} T-T^{2}+\left(2 R_{0}^{\prime}-T\right)\left(\cos \theta-T \cos ^{2} \theta\right)\right]}\right] .
\end{aligned}
$$

where

$$
\begin{aligned}
C_{1}= & -48\left[1+\left(E_{m}^{2}-1\right) A^{2}\right] R_{0}^{\prime} \\
& +8\left[3\left(3 E_{m}^{2}+1\right)-\left(5 E_{m}^{2}+3\right) A^{2}+3\left(E_{m}^{2}+4\right) A R_{0}^{\prime}-4\left(E_{m}^{2}+3\right) A^{3} R_{0}^{\prime}\right] T \\
& +4\left[4\left(E_{m}^{2}+3\right) A^{3}-\left(E_{m}^{2}+12\right) A+6\left(E_{m}^{2}-2\right) R_{0}^{\prime}+12 A^{2} R_{0}^{\prime}\right] T^{2} \\
& +2\left[4\left(2 E_{m}^{2}-3\right) A^{2}-2\left(i E_{m}^{2}-6\right)+3 E_{m}^{2} A R_{0}^{\prime}\right] T^{3}-E_{m}^{2}\left(5 A+R_{0}^{\prime}\right) T^{4} \\
C_{2}= & -16\left(9 E_{m}^{2}-1\right)\left(1-A R_{0}^{\prime}\right) \\
& +8\left[3\left(E_{m}^{2}-1\right) A-\left(18 E_{m}^{2}+1\right) R_{0}^{\prime}+4\left(3 E_{m}^{2}+1\right) A^{2} R_{0}^{\prime}\right] T \\
& +4\left[21 E_{m}^{2}+4-4\left(3 E_{m}^{2}+1\right) A^{2}-12 E_{m}^{2} A R_{0}^{\prime}\right] T^{2} \\
& +2\left[4 A+\left(3 E_{m}^{2}-4\right) R_{0}^{\prime}\right] T^{3}+3 E_{m}^{2} T^{4}, \\
C_{3}= & -24\left(E_{m}^{2}-1+R_{0}^{\prime 2}\right) R_{0}^{\prime} \\
& -4\left[5 E_{m}^{2}+3+4\left(E_{m}^{2}+3\right) A R_{0}^{\prime}-3\left(1+4 A R_{0}^{\prime}\right) R_{0}^{2}\right] T \\
& +2\left[4\left(E_{m}^{2}+3\right) A+\left(11 E_{m}^{2}+12\right) R_{0}^{\prime}-12\left(A+R_{0}^{\prime}\right) R_{0}^{\prime 2}\right] T^{2} \\
& +\left(5 E_{m}^{2}-12+4 E_{m}^{2} A R_{0}^{\prime}+12 R_{0}^{\prime 2}\right) T^{3}-2 E_{m}^{2}\left(A+2 R_{0}^{\prime}\right) T^{4} \\
C_{4}= & -32\left(1-A R_{0}^{\prime}\right) R_{0}^{\prime}+4\left(3 E_{m}^{2}+5-5 R_{0}^{\prime 2}\right) T-8\left(A-R_{0}^{\prime}\right) T^{2}-3 E_{m}^{2} T^{3}, \\
D= & -24\left(1-A R_{0}^{\prime}\right) R_{0}^{\prime}+8\left[2-A R_{0}^{\prime}-\left(3-2 A^{2}\right) R_{0}^{\prime 2}\right] T \\
& -2\left[A-\left(13-8 A^{2}\right) R_{0}^{\prime}+4 A R_{0}^{\prime 2}\right] T^{2}-\left(9-4 A^{2}-4 A R_{0}^{\prime}-R_{0}^{\prime 2}\right) T^{3} .
\end{aligned}
$$

Integration over the poloidal angle $\theta$ gives

$$
\begin{aligned}
\mu_{0} E_{m} K(\rho) \cong & \frac{2\left(E_{m}^{2}-1\right)}{-R_{0}^{\prime}+T / 2}+\frac{C_{1}+C_{2} \sqrt{A^{2}-1}}{2 D \sqrt{A^{2}-1} \sqrt{1-T\left(A-\sqrt{A^{2}-1}\right)}} \\
& +\frac{\left(1-T^{2} / 4\right)\left[C_{3}+2 C_{4} \sqrt{\left(1-R_{0}^{\prime 2}\right)\left(1-T^{2} / 4\right)}\right.}{\left(-R_{0}^{\prime}+T / 2\right) D \sqrt{\left(1-R_{0}^{\prime 2}\right)\left(1-T^{2} / 4\right)}} \\
& \times\left[\frac{-R_{0}^{\prime}+T / 2}{-R_{0}^{\prime}\left(1-T^{2}\right)-3 T / 2+2 T \sqrt{\left(1-R_{0}^{\prime 2}\right)\left(1-T^{2} / 4\right)}}\right]^{1 / 2} .
\end{aligned}
$$


One verifies that $L(\rho)$ and $K(\rho$.) are real if the previous conditions involving $A, T$ and $R_{0}^{\prime}$ are satisfied. Taking the limit $T \rightarrow 0$ yields for an elliptical plasma cross-section

$$
\begin{aligned}
\mu_{0} E_{m} K(\rho) \underset{T \rightarrow 0}{\rightarrow} & \frac{1}{1-A R_{0}^{\prime}}\left[\frac{1+\left(E_{m}^{2}-1\right) A^{2}}{\sqrt{A^{2}-1}}+\frac{-R_{0}^{\prime}+\left(E_{m}^{2}-1\right) /\left(-R_{0}^{\prime}\right)}{\sqrt{1-R_{0}^{\prime 2}}}\right] \\
& -\frac{E_{m}^{2}-1}{\left(-R_{0}^{\prime}\right)} \underset{\rho \rightarrow 0}{\rightarrow}\left(\frac{E_{m}^{2}+1}{2}\right) \frac{\rho}{R_{m}} .
\end{aligned}
$$

Using the limiting values of $L(\rho)$ and $K(\rho)$ the value of the safety factor on the magnetic axis becomes

$$
q(0)=\frac{\left(E_{m}^{2}+1\right) B_{m}}{\mu_{0} E_{m} R_{m}} \lim _{\rho-0}\left(\frac{\pi E_{m} \rho^{2}}{I_{T}(\rho)}\right)=\frac{\left(E_{m}^{2}+1\right) B_{m}}{\mu_{0} E_{m} R_{m} j_{T}(0)} .
$$

The derivatives $d V / d \rho$ and $d L / d \rho$ can be calculated directly. Alternatively, one may resort to integrations according to the definitions

$$
\begin{aligned}
& \frac{d V}{d \rho}=2 \pi \int_{0}^{2 \pi} R\left(\frac{\partial R}{\partial \rho} \frac{\partial Z}{\partial \theta}-\frac{\partial R}{\partial \theta} \frac{\partial Z}{\partial \rho}\right) d \theta \\
& \frac{d L}{d \rho}=\frac{\mu_{0}}{2 \pi} \int_{0}^{2 \pi} \frac{1}{R}\left(\frac{\partial R}{\partial \rho} \frac{\partial Z}{\partial \theta}-\frac{\partial R}{\partial \theta} \frac{\partial Z}{\partial \rho}\right) d \theta .
\end{aligned}
$$

Finally, one may point out that the coefficients in the flux-surface averaged equilibrium equation can be calculated analytically for trial functions of arbitrary dependence on the topological radius and for an arbitrary number of terms in the Fourier series expansions $R(\rho, \theta)$ and $Z(\rho, \theta)$, as will be shown in a future paper. This effectively reduces the solution of the variational problem, either by the direct method described here or by the moment method [7], to a one-dimensional problem. The discussion in the present paper is restricted to a particularly simple model that leads to explicit expressions for the equilibrium coefficients in terms of algebraic functions.

\section{Examples and Discussion}

For the geometry under consideration the two-dimensional fixed boundary equilibrium problem has been reduced, in accordance to the Ritz procedure, 
to the optimization of a single parameter which is the position $R_{m}$ of the magnetic axis. The radial integration in the functional $U(a)$ has to be performed numerically. It depends on the profiles specified for the plasma pressure $p(\rho)$ and the toroidal current $I_{T}(\rho)$ which are here assumed of the form

$$
\begin{aligned}
p(\rho) & =p(0)\left[1-(\rho / a)^{2}\right]^{\alpha_{p}} \\
I_{T}(\rho) & =I_{T}(a)\left(\frac{\rho}{a}\right)^{2}\left[\left(1+\frac{1}{\alpha_{I}}\right)-\frac{1}{\alpha_{I}}\left(\frac{\rho}{a}\right)^{2}\right]^{\alpha_{I}} .
\end{aligned}
$$

The model described in this paper was implemented using the Mathematica package [10]. The search for stationary points of the internal energy $U(a)$ as a function of $R_{m}$ can be easily carried out using the numerical minimization functions provided by Mathematica. In order to illustrate the method, the results of two different tokamak configurations are presented: (1) an equilibrium solution for the small-aspect-ratio NSTX tokamak and (2) an equilibrium solution for the advanced TPX tokamak. The parameters for these two configurations are listed in table 1. In both cases approximately parabolic profiles are assumed with $\alpha_{p}=2$ and $\alpha_{I}=1 / 2$. The peak pressure $p(0)$ listed in table 1 corresponds to a value $q_{0}=1$ of the safety factor on the magnetic axis for the small-aspect-ratio configuration. The standardaspect-ratio equilibrium represented by TPX corresponds to a beta value of approximately $6 \%$. The plasma beta is the ratio between the thermal energy and the magnetostatic energy of the vacuum field in the plasma region

$$
\beta=\frac{W_{t h}(a)}{W_{T, 0}(a)} .
$$

The toroidal beta and the average plasma pressure are defined by:

$$
\begin{aligned}
& \beta_{T, 0}=\frac{3 \bar{p}(a) / 2}{B_{0}^{2} /\left(2 \mu_{0}\right)} . \\
& \bar{p}(a)=\frac{2}{3} \frac{W_{t h}(a)}{V(a)} .
\end{aligned}
$$

Figure 1 shows the flux-surface contours for the NSTX equilibrium and figure 2 shows the flux-surface averaged toroidal plasma current density $j_{T}(\rho)$ and safety factor $q(\rho)$ profiles. The poloidal and toroidal components 


\begin{tabular}{|l|c|c|}
\hline & NSTX & TPX \\
\hline \hline Major radius $R_{0}(a)[\mathrm{m}]$ & 0.80 & 2.25 \\
Minor radius $a[\mathrm{~m}]$ & 0.55 & 0.50 \\
Elongation $\kappa(a)$ & 1.6 & 1.7 \\
Triangularity $\delta(a)$ & 0.4 & 0.4 \\
External toroidal induction $B_{0}[\mathrm{~T}]$ & 0.5 & 4.0 \\
Toroidal plasma current $I_{T}(a)[\mathrm{MA}]$ & 0.9 & 2.0 \\
Pressure on the magnetic axis $p(0)[\mathrm{MPa}]$ & 0.010 & 1.2 \\
Internal inductance $\ell_{i}$ & 0.54 & 0.65 \\
Current diamagnetism $\mu_{I}$ & 0.51 & -1.58 \\
Current beta $\beta_{I}$ & 0.11 & 2.52 \\
Plasma beta $\beta$ & 0.028 & 0.062 \\
Toroidal beta $\beta_{T, 0}$ & 0.038 & 0.065 \\
Safety factor on the magnetic axis $q(0)$ & 1.00 & 1.06 \\
Safety factor at the plasma edge $q(a)$ & 5.5 & 2.7 \\
\hline
\end{tabular}

Table 1: Equilibrium parameters of the NSTX and TPX equilibria

$j_{P}(R, 0), j_{T}(R, 0)$ of the plasma current density, respectively, and $B_{P}(R, 0)$, $B_{T}(R, 0)$ of the induction on the horizontal midplane $(Z=0)$, are shown in figure 3. The same sequence of contours and profiles are shown in figures 4,5 and 6 for the TPX equilibrium. One verifies the slightly paramagnetic nature of the small-aspect-ratio equilibrium near the pressure limit.

The calculations where carried out using a PC type computer. The radial integrations were performed using Gaussian quadrature with as few as three points to obtain two to three digits precision in the results. One expects that the simple model presented is sufficiently accurate to represent the equilibrium of $D$-shaped tokamak plasmas. The set of trial functions can be improved by allowing radial variation of the elongation coefficient. However, this implies introducing quadrangularity effects in order to get a consistent expansion of the trial functions to fourth order near the magnetic axis, according to the procedure outlined in section 3 . In this way, the list of free parameters to be optimized increases with the inclusion of the elongation $E_{m}$ on the magnetic axis. Finally, one may point out that the direct method can be easily applied to find equilibrium solutions consistent, for example, with experimentally measured profiles of the plasma pressure. 


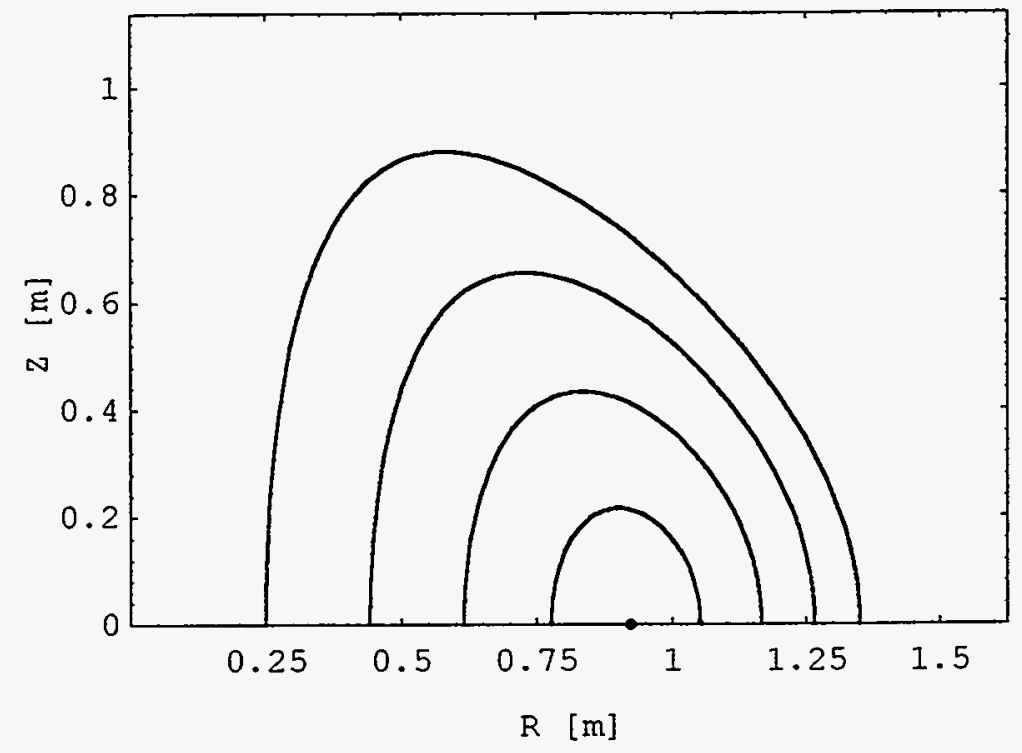

Figure 1: Magnetic flux surfaces contours for the NSTX small-aspect-ratio tokamak equilibrium.
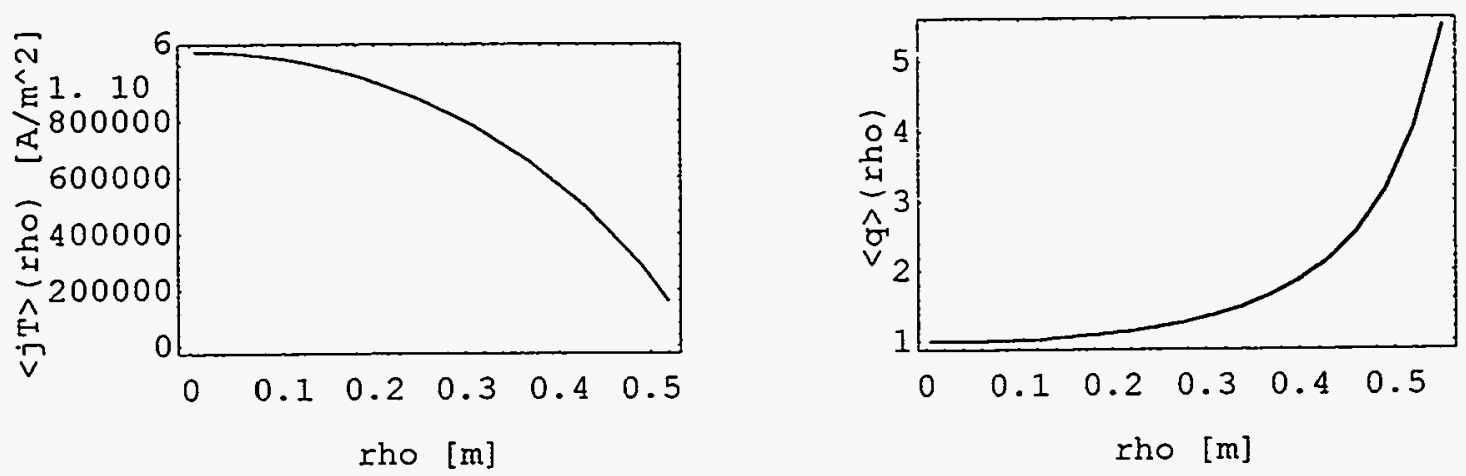

Figure 2: Flux-surface averaged toroidal plasma current density and safety factor profiles for the NSTX equilibrium. 

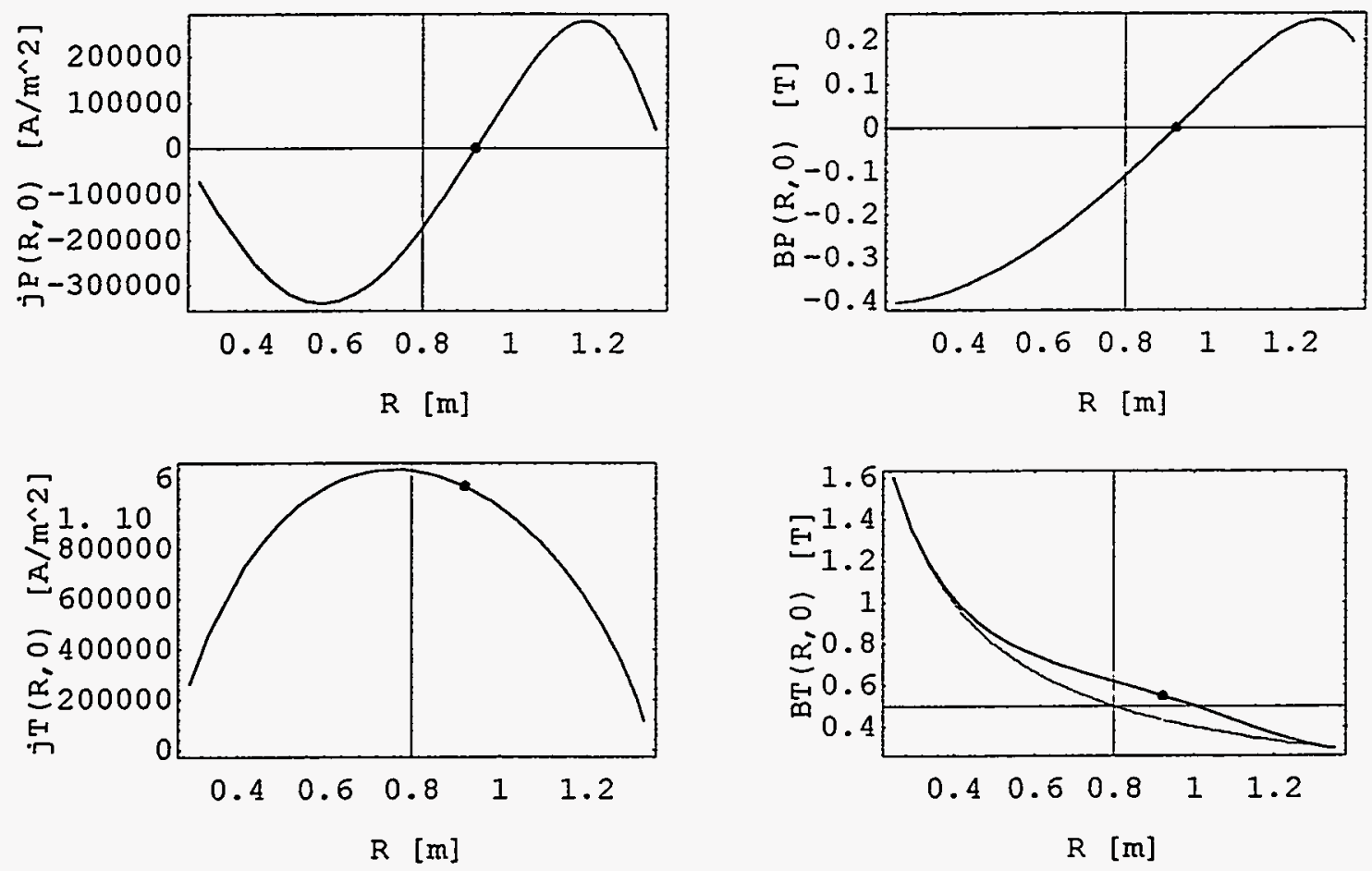

Figure 3: Poloidal and toroidal components of the plasma current density and induction on the horizontal midplane for the NSTX equilibrium. The point indicates the position of the magnetic axis and the curve in gray corresponds to the external induction. 


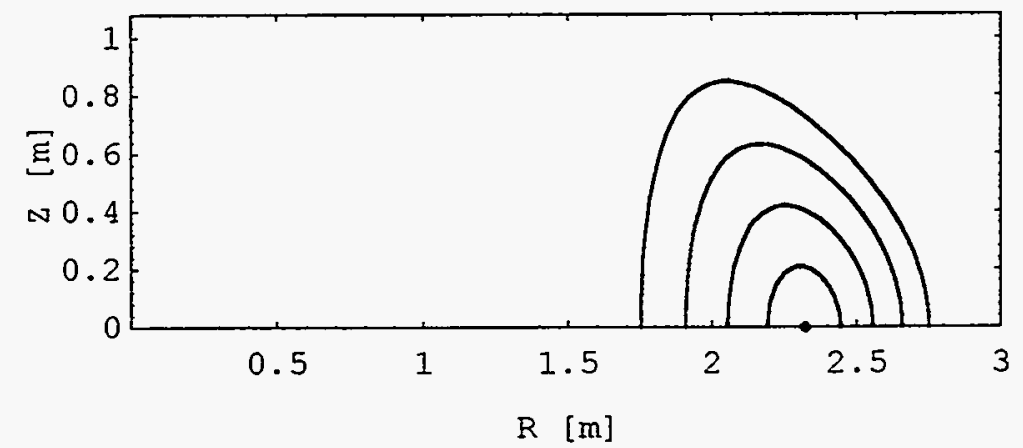

Figure 4: Magnetic flux surfaces contours for the TPX advanced tokamak equilibrium.
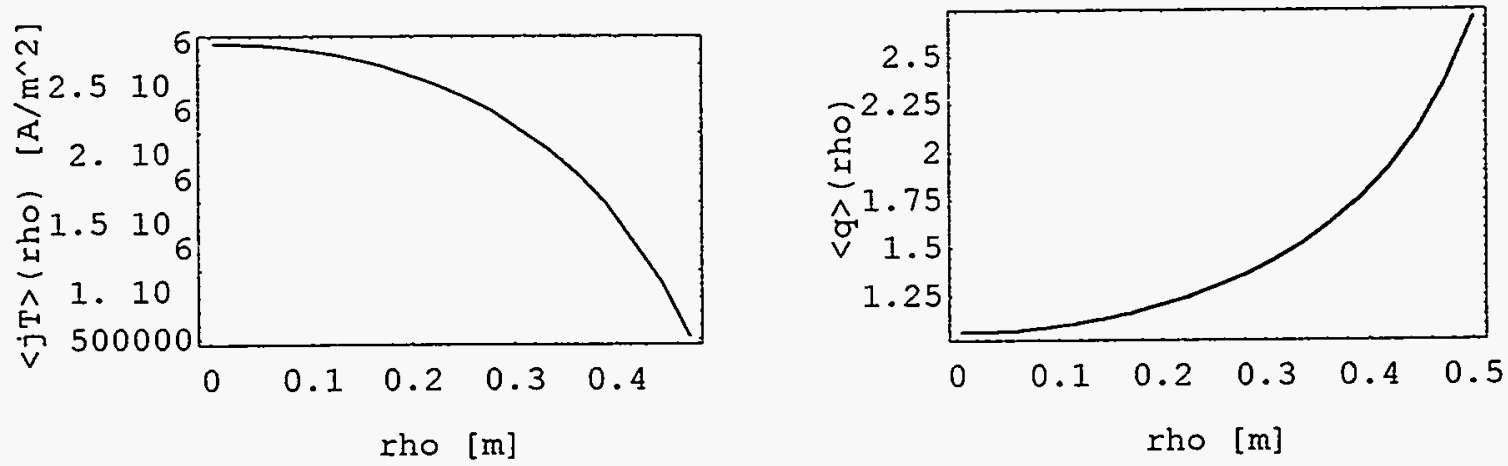

Figure 5: Flux-surface averaged toroidal plasma current density and safety factor profiles for the TPX equilibrium. 

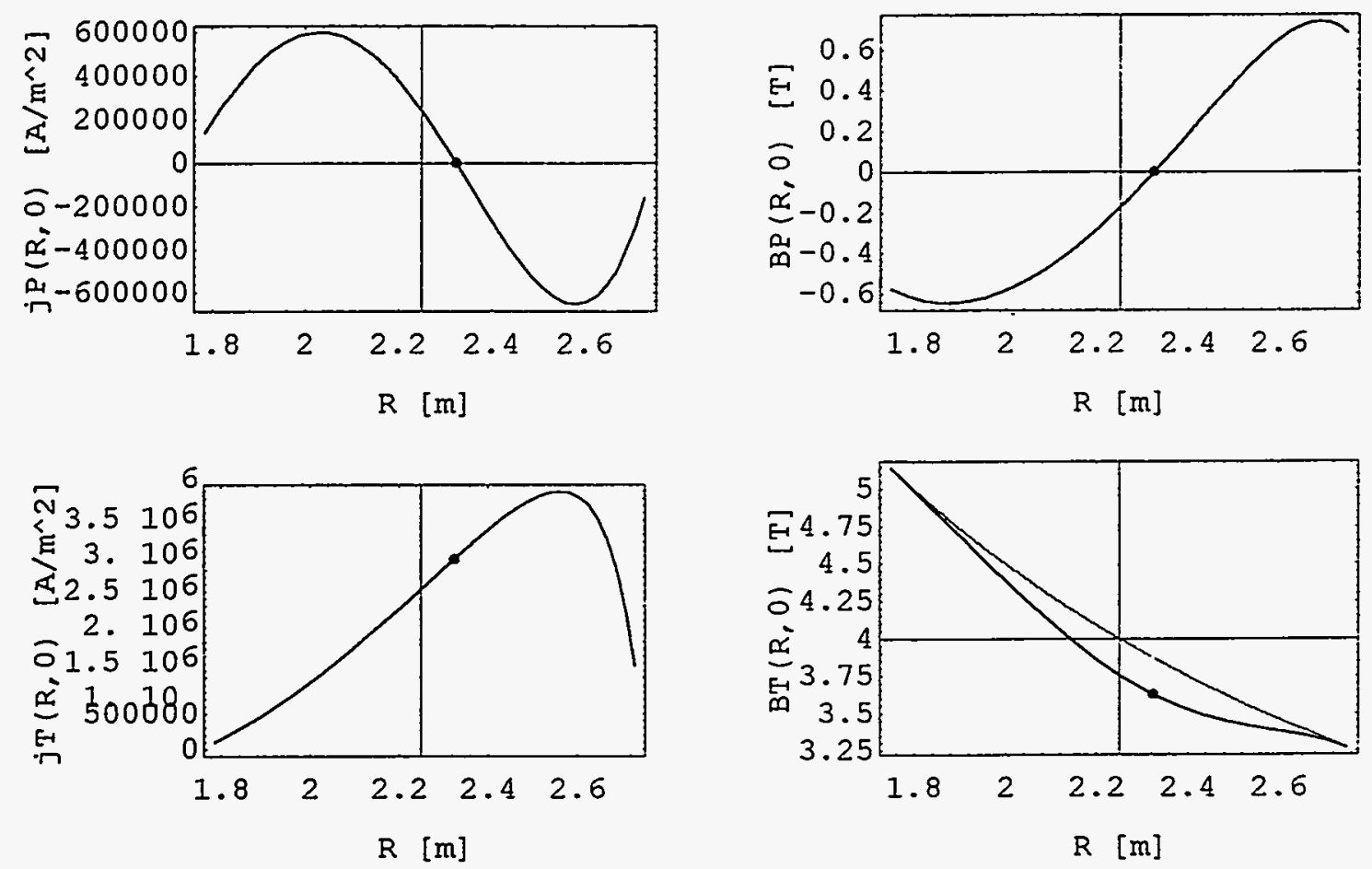

Figure 6: Poloidal and toroidal components of the plasma current density and induction on the horizontal midplane for the TPX equilibrium. The point indicates the position of the magnetic axis and the curve in gray corresponds to the external induction. 
Acknowledgment: This work has been supported under Department of Energy contract NO DE-AC02-76-CHO-3073. 


\section{References}

[1] Kruskal M D and Kulsrud R M 1958 Phys. Fluids 1265

[2] Grad H 1964 Phys. Fluids 71283

[3] Lüst R and Schlüter A 195ॅ Z. Naturforschg. 12a 85

[4] Shafranov V D 1958 Sov. Phys. JETP 6.545

[5] Grad H and Rubin H 1958 Proceedings of the Second United Nations International Conference on the Peaceful Uses of Atomic Energy (Geneva: United Nations) vol 31 p 190

[6] Khait V D 1980 Sov. J. Plasma Physics 6476

[7] Lao L L, Hirshman S P and Wieland R M 1981 Phys. Fluids 241431

[8] Zhakharov L E and Shafranov V D 1986 Reviews of Plasma Physics, edited by M A Leontovich (New York: Consultants Bureau) vol $11 \mathrm{p}$ 153

[9] Weitzner H 1981 appendix in reference 7

[10] Mathematica 1992 (Champaign, Illinois: Wolfram Research, Inc.) version 2.2 


\section{$6 \quad$ List of Figures}

Figure 1: Magnetic flux surfaces contours for the NSTX small-aspect-ratio tokamak equilibrium.

Figure 2: Flux-surface averaged toroidal plasma current density and safety factor profiles for the NSTX equilibrium.

Figure 3: Poloidal and toroidal components of the plasma current density and induction on the horizontal midplane for the NSTX equilibrium. The point indicates the position of the magnetic axis and the curve in gray corresponds to the external induction.

Figure 4: Magnetic flux surfaces contours for the TPX advanced tokamak equilibrium.

Figure 5: Flux-surface averaged toroidal plasma current density and safety factor profiles for the TPX equilibrium.

Figure 6: Poloidal and toroidal components of the plasma current density and induction on the horizontal midplane for the TPX equilibrium. The point indicates the position of the magnetic axis and the curve in gray corresponds to the external induction. 
Dr. F. Paoloni, Univ. of Wollongong, AUSTRALIA

Prof. R.C. Cross, Univ. of Sydney, AUSTRALIA

Plasma Research Lab., Australian Nat. Univ., AUSTRALIA

Prof. I.A. Jones, Flinders Univ, AUSTRALIA

Prof. F. Cap, Inst. for Theoretical Physics, AUSTRIA

Prof. M. Heindler, Institut für Theoretische Physik, AUSTRIA •

Prof. M. Goossens, Astronomisch Instituut, BELGIUM

Ecole Royale Militaire, Lab. de Phy. Plasmas, BELGIUM

Commission-European, DG. XII-Fusion Prog., BELGIUM

Prol. R. Bouciqué, Rijksuniversiteit Gent, BELGIUM

Dr. P.H. Sakanaka, Instituto Fisica, BRAZIL

Prof. Dr. I.C. Nascimento, Instituto Fisica, Sao Paulo, BRAZIL Instituto Nacional De Pesquisas Espaciais-INPE, BRAZIL

Documents Office, Atomic Energy of Canada Lid., CANADA

Ms. M. Morin, CCFMTokamak de Varennes, CANADA

Dr. M.P. Bachynski, MPB Technologies, Inc., CANADA

Dr. H.M. Skarsgard, Univ. of Saskatchewan, CANADA

Prof. J. Teichmann, Univ. of Montreal, CANADA

Prof. S.R. Sreenivasan, Univ. of Calgary, CANADA

Prof. T.W. Johnston, INAS-Energie, CANADA

Dr. R. Bolton, Centre canadien de fusion magnétique, CANADA

Dr. C.R. James, Univ. of Alberta, CANADA

Dr. P. Lukác, Komenského Universzita, CZECHO-SLOVAKIA

The Librarian, Culham Laboratory. ENGLAND

Library, R61, Rutherford Appleton Laboratory, ENGLAND

Mrs. S.A. Hutchinson, JET Library, ENGLAND

Dr. S.C. Sharma, Univ. of South Pacific, FIJI ISLANDS

P. Mähönen, Univ. of Helsinki, FINLAND

Prof. M.N. Bussac, Ecole Polytechnique, FRANCE

C. Mouttet, Lab. de Physique des Milieux lonisés, FRANCE

J. Radet, CEN/CADARACHE - Bat 506, FRANCE

Prof. E. Economou, Univ. of Crete, GREECE

Ms. C. Rinni, Univ. of loannina, GREECE

Preprint Library, Hungarian Academy of Sci., HUNGARY

Dr. B. DasGupta, Saha Inst. of Nuclear Physics, INDIA

Dr. P. Kaw, Inst. for Plasma Research, INDIA

Dr. P. Rosenau, Israel Inst. of Technology, ISRAEL

Librarian, International Center for Theo Physics, ITALY

Miss C. De Palo, Associazione EURATOM-ENEA, ITALY

Dr. G. Grosso, Istituto di Fisica del Plasma, ITALY

Prof. G. Rostangni, Istituto Gas lonizzati Del Cnr, ITALY
Dr. H. Yamato, Toshiba Res \& Devel Center, JAPAN

Prof. I. Kawakami, Hiroshima Univ., JAPAN

Prof. K. Nishikawa, Hiroshima Univ., JAPAN

Librarian, Naka Fusion Research Establishment, JAERI, JAPAN

Director, Japan Atomic Energy Research Inst., JAPAN

Prof. S. Itoh, Kyushu Univ., JAPAN

Research Info. Ctr., National Instit. for Fusion Science, JAPAN

Prof. S. Tanaka, Kyoto Univ., JAPAN

Library, Kyoto Univ., JAPAN

Prof. N. Inoue, Univ. of Tokyo, JAPAN

Secretary, Plasma Section, Electrotechnical Lab., JAPAN

Dr. O. Mitarai, Kumamoto Inst. of Technology, JAPAN

Dr. G.S. Lee, Korea Basic Sci. Crr., KOREA

J. Hyeon-Sook, Korea Atomic Energy Research Inst., KOREA

D.I. Choi, The Korea Adv. Inst. of Sci. \& Tech., KOREA

Prof. B.S. Liley, Univ. of Waikato, NEW ZEALAND

Inst of Physics, Chinese Acad Sci PEOPLE'S REP. OF CHINA

Library, Inst. of Plasma Physics, PEOPLE'S REP. OF CHINA

Tsinghua Univ. Library, PEOPLE'S REPUBLIC OF CHINA

Z. Li, S.W. Inst Physics, PEOPLE'S REPUBLIC OF CHINA

Prof. J.A.C. Cabral, Instituto Superior Tecnico, PORTUGAL

Prof. M.A. Hellberg, Univ. of Natal, S. AFRICA

Prof. D.E. Kim, Pohang Inst. of Sci. \& Tech., SO. KOREA

Prof. C.I.E.M.A.T, Fusion Division Library, SPAIN

Dr. L. Stenflo, Univ. of UMEA, SWEDEN

Library, Royal Inst. of Technology, SWEDEN

Prof. H. Wilhelmson, Chalmers Univ. of Tech., SWEDEN

Centre Phys. Des Plasmas, Ecole Polytech. SWITZERLAND

Bibliotheek, Inst. Voor Plasma-Fysica, THE NETHERLANDS

Asst. Prof. Dr. S. Cakir, Middle East Tech. Univ., TURKEY

Dr. V.A. Glukhikh,Sci. Res. Inst. Electrophys.I Apparatus, USSR

Dr. D.D. Ryutov, Siberian Branch of Academy of Sci., USSR

Dr. G.A. Eliseev, I.V. Kurchatov Inst., USSA

Librarian, The Ukr.SSR Academy of Sciences, USSR

Dr. L.M. Kovrizhnykh, Inst. of General Physics, USSR

Kemforschungsanlage GmbH, Zentralbibliothek, W. GERMANY Bibliothek, Inst. Für Plasmaforschung, W. GERMANY

Prof. K. Schindler, Ruhr-Universitát Bochum, W. GERMANY

Dr. F. Wagner, (ASDEX), Max-Planck-Institut, W. GERMANY

Librarian, Max-Planck-Institut, W. GERMANY 
